\title{
MedienPädagogik
}

Zeitschrift für Theorie und Praxis der Medienbildung

Themenheft Nr. 36: Teilhabe in einer durch digitale Medien geprägten Welt Perspektiven des wissenschaftlichen Nachwuchses

Herausgegeben von Tim Riplinger, Jan Hellriegel und Ricarda Bolten

\section{Editorial: Teilhabe in einer durch digitale Medien geprägten Welt}

\section{Perspektiven des wissenschaftlichen Nachwuchses}

Tim Riplinger, Jan Hellriegel und Ricarda Bolten

Das Junge Forum für Medien und Hochschulentwicklung (JFMH)

Vom 19. bis 20. Juli 2018 durften wir die Teilnehmenden des Jungen Forums für Medien und Hochschulentwicklung (JFMH) an der TU Kaiserslautern begrüssen. Bei schönstem Sommerwetter verbrachten wir zwei Tage mit konstruktivem Austausch in verschiedenen Formaten und geselligem Beisammensein. Hierbei zeigte sich vor allem der positive Grundgedanke für den wissenschaftlichen Nachwuchs: Erfahrung trifft Neugier, konstruktiver Austausch und Vernetzung. Das ist das Ziel des JFMH: Jungen Wissenschaftlerinnen und Wissenschaftlern die Möglichkeit zu geben in einem geschützten Raum frühe Erfahrungen bei der Präsentation und Diskussion ihrer Forschungsprojekte zu ermöglichen. Wissenschaftlerinnen und Wissenschaftlern in der Qualifizierungsphase (Young Researcher) und junge Wissenschaftlerinnen und Wissenschaftler, die an praxisnahen Forschungs- oder Entwicklungsprojekten beteiligt sind (Young Professionals) kommen dabei zusammen, um gemeinsam Themen zu Medien und Hochschuldidaktik oder verwandten Bereichen zu diskutieren und sich dazu auszutauschen. Begleitet werden die Beiträge durch die Deutsche Gesellschaft für Hochschuldidaktik (dghd), die Gesellschaft für Informatik (GI, Fachgruppe E-Learning), die Gesellschaft für Medien in der Wissenschaft (GMW) und die Deutsche Gesellschaft für Erziehungswissenschaft (DGfE, Sektion Medienpädagogik), die seit 2012 jährlich gemeinsam mit den jeweiligen ausrichtenden Institutionen das JFMH gestalten. Das JFMH 2018 widmete sich dem Thema «Teilhabe in einer durch digitale Medien geprägten Welt», welches auch zentrales Thema dieses Tagungsbandes ist, der als Heft 36 bei der Zeitschrift MedienPädagogik erscheint. Durch die nun schon sechsjährige Tradition des JFMHs nehmen auch viele erfahrene Wissenschaftlerinnen und Wissenschaftler an der Tagung teil, die häufig selbst frühe Erfahrungen auf der JFMH gesammelt haben. Diese Zusammenkunft verschiedener Stadien in der Wissenschaft und wissenschaftlichen Austausches brachte auch 2018 wieder eine sehr produktive und gesellige Atmosphäre hervor.

Die Vortragenden und auch die Autorinnen und Autoren, die in diesem Tagungsband ihre Beiträge präsentieren, wurden während des gesamten Prozesses im Rahmen eines Peer-Reviews begleitet. Im Rahmen dieses offenen Review-Verfahrens wird den Reviewerinnen und Reviewern sowie den Vortragenden/Autorinnen und 
Autoren die Möglichkeit geboten im kompletten Review-Prozess in einem gegenseitigen Austausch die Beiträge qualitativ weiter zu stärken. Auch dies unterstreicht das Ziel des JFMH: Jungen Wissenschaftlerinnen und Wissenschaftlern die Möglichkeit zu bieten, sich in einem konstruktiven Vorgang an die Prozesse des Präsentieren und Veröffentlichen eigener Forschungsergebnisse anzunähern. Die Unterstützung erfahrener Wissenschaftlerinnen und Wissenschaftlern aus der Fachcommunity erweist sich seit Jahren als sehr fruchtbar.

Bevor das Hauptprogramm des JFMH 2018 startete, gab es zwei parallele Angebote zur Auswahl. Zum einen, thematisch passend, einen runden Tisch „OER in der Region" mit einer Diskussion um den Mehrwert offener digitaler Bildung(-smaterialien) und Bildungspraktiken sowie die Frage danach, wie Bürger*innen Teilhabe an Bildungsprozessen durch digitale Medien und freie Bildungsmaterialien ermöglicht werden kann. Diese Veranstaltung war Teil des Projekts OERlabs der Universität zu Köln und der TU Kaiserslautern. Zum anderen wurde von Matthias Rohs ein Workshop zu Social Media in der Forschung angeboten und der Frage nachgegangen, ob diese eine Chance zur Vernetzung oder doch nur Ablenkung seien?

Nach der Begrüssung des Organisations-Teams und einer Keynote von Patrick Bettinger («Mit Medien etwas machen oder das Mitmachen der Medien? Teilhabe und verteiltes Handeln in sozio-medialen Kollektiven») ging es zu den parallel stattfinden Vortragstracks. Dabei kam der erste von insgesamt vier flipped conference-Beiträgen zum Einsatz, d.h. dass die Präsentation des Themas im Vorfeld in Videoform bereitgestellt wurde und die kostbare Zeit auf der Tagung für intensive Diskussionen genutzt werden konnte. Im Anschluss fand die Posterpräsentation im Foyer statt und da keine parallelen Vorträge geplant waren, bekamen die Poster zurecht die ungeteilte Aufmerksamkeit des gesamten Tagungspublikums. Bevor das gesellige Abendprogramm starten konnte, wartete noch ein 90-minütiges World Café auf die Teilnehmenden, welches das Tagungsthema aufgriff und die Frage stellte, wie Teilhabe ermöglicht und nachhaltig gesichert werden kann. Nach diesem letzten inhaltlichen Slot wurde bei bestem Wetter, Grill und Kaltgetränken der erste Tag abgerundet und gemeinsam abgeschlossen.

Tag zwei begann mit einer Keynote von Claudia Bremer, die die Herausforderungen und Chancen beim Thema Teilhabe und digitale Medien für Hochschulen und Universitäten darlegte und dabei die Entwicklungen der letzten Jahre aufzeigte. Im Anschluss hielt Felix Fischer die Nachwuchskeynote und stellte sich die Frage, wie Design als „Erzieher*in“ die menschliche Konstitution beeinflusst und wie Imagination, als Erkenntnis- und Bildungsprozess, dazu beitragen kann, Mündigkeit und damit Teilhabe zu sichern. Nach zwei intensiven Sessions mit jeweils zwei parallelen Tracks folge eine kurze Zusammenfassung von Seiten des Organisationsteams mit einigen Danksagungen und schliesslich eine herzliche Verabschiedung. 
Teilhabe in einer durch digitale Medien geprägten Welt als Leitthema der Tagung Gesellschaftliche Teilhabe lässt sich als ein grundlegendes Ziel von Bildungsprozessen verstehen. Da digitale Medien in der heutigen Zeit die Lebens- und Arbeitswelt immer stärker durchdringen, wird an vielen Stellen über grundlegende und barrierearme Medienbildung als Voraussetzung zur Selbstbestimmung und gesellschaftlichen Teilhabe diskutiert. Im Zuge der Digitalisierung finden Prozesse der Kommunikation, der Informationsbeschaffung und der Partizipation an Politik, Wirtschaft und Gesellschaft zunehmend in virtuellen Welten statt. Digitale Medien bieten dabei grundsätzlich die Chance einer zeit- und ortsunabhängigen Kommunikation sowie eines räumlich und zeitlich entkoppelten Lernens.

Wer jedoch nicht über ausreichende Medienkompetenz oder nicht über die erforderliche technische Ausstattung und Infrastruktur verfügt, läuft Gefahr, am beruflichen und gesellschaftlichen Leben nicht gleichberechtigt partizipieren zu können. Hiervon sind neben Personen mit inklusivem Förderbedarf beispielsweise auch Personen in ländlich geprägten Räumen betroffen, beispielsweise aufgrund fehlender Breitbandverbindungen. Aktuelle Phänomene wie «Fake News», «Social Bots» oder «Filter Bubbles» zeigen zudem, dass Medien auch Risiken im Hinblick auf gesellschaftliche Teilhabe sein können, da durch solche Entwicklungen ein verzerrtes Bild von Gesellschaft entstehen kann.

Daher ist es gerade im Kontext von Lehre und Forschung sowie in Qualifizierungsphasen von Studierenden und Promovierenden bedeutsam, sich reflektiert und differenziert mit den sich wandelnden Herausforderungen, aber auch den gegebenen Chancen, die digitale Medien im Hinblick auf Teilhabe bieten, zu befassen. Teilhabe muss dabei interdisziplinär gedacht werden, so dass Sichtweisen aus verschiedenen gesellschaftlichen Kontexten und Fachlogiken zusammengeführt werden können. Aufgrund der Bedeutsamkeit dieser Thematik befasste sich das JFMH 2018 mit dem Thema Teilhabe in einer durch digitale Medien geprägten Welt.

Im Rahmen der Tagung eröffneten sich verschiedene Zugänge zu diesem Thema. Zum Beispiel gingen die Teilnehmenden im Rahmen des World Cafés der Frage nach, wie Teilhabe ermöglicht und nachhaltig gesichert werden kann und welche Anforderungen damit einhergehen. Dabei wurden fünf Teilaspekte identifiziert, zu denen rege diskutiert wurde:

- Anforderungen an die technischen/medialen Rahmenbedingungen

- Anforderungen an das Personal/die Lehrenden (und deren Grundhaltungen) sowie an die Personalentwicklung

- Anforderungen an die organisatorischen Rahmenbedingungen

- Anforderungen an die pädagogisch-didaktischen Konzepte

- Anforderungen an die Lernenden 
In den Session-Vorträgen und Posterbeiträgen wurde Teilhabe auch aus einer demokratischen Perspektive (Heike Wehage) thematisiert oder auch im Hinblick auf studentische Partizipation (Wolfgang Ruge, Gerhard Pölsterl, Daniel Haslinger und Wolfram Grosser) sowie im Hinblick auf die digitale Teilhabe von Kindern und den Anforderungen an die Eltern - insbesondere im Hinblick auf die medienpädagogische Facette der Medienkritikfähigkeit (Karin Rott).

Die Beiträge in diesem Themenheft

Nachfolgend werden die am Tagungsband beteiligten Beiträge im Einzelnen vorgestellt.

Felix Fischer leistet in seinem Beitrag «Imagination by Design» einen Klärungsversuch, wie Design konstituiert wird und an welchen Punkten Bildung ansetzen muss, um ein selbstbestimmtes Agieren in designten Welten sicherzustellen. Mit Verweis auf die Idee von ‘Imagineering) wird vorgeschlagen, Design als mentales Image zu konzeptualisieren, um ein Verständnis davon zu erlangen, wie Design-Images verändert werden können, ohne dabei die materiellen oder sensorischen Elemente zu verändern.

Eva-Maria Glade und Michael Schön stellen in ihrem Beitrag "Reflexive Professionalisierung von Schulleitungshandeln» zunächst die veränderten Aufgaben an Schulleitungen vor, die sich aus der Digitalisierung ergeben und gehen ferner auf die dafür notwendigen emotionalen, sozialen und transformativen Führungskompetenzen ein. Abschliessend wird ein Einblick in ein Medienbildungsmodul eines Fernstudienangebots zur Fort- und Weiterbildung pädagogischer Führungskräfte gegeben.

Der Beitrag «Das Märchen der Digital Natives» von Kim Lucia Deutsch und Sebastian Kuhn widmet sich der Frage, wie Hochschulbildung den digitalen Wandel adressieren, begleiten und Studierende so zur beruflichen Kompetenz und aktiven gesellschaftlichen Teilhabe befähigen kann. Dabei werden Bezüge zum Lehrkonzept «Medizin im digitalen Zeitalter» hergestellt und Evaluationsergebnisse vorgestellt.

Imke Buß und Anne Keller beschäftigen sich in ihrem Beitrag mit virtuellen Lehren und Lernen an der Hochschule für Wirtschaft und Gesellschaft in Ludwigshafen. Sie stellen Ergebnisse einer Untersuchung mit Hochschullehrenden in Bezug auf digitale Kompetenzen und die eigenen Erfahrungen mit virtueller Lehre sowie die Motivation der Lehrenden für den Einsatz von E-Teaching vor.

Fit für die digitale Hochschule? Diese Frage befasst Michael Eichhorn in seinem gleichnamigen Beitrag, in dem er die Entwicklung eines Kompetenzmodells beschreibt, mit dem sich digitale Kompetenzen von Hochschullehrenden erfassen und vergleichen lassen. Darauf aufbauend beschreibt er ein auf diesem Modell basierendes Kompetenzraster sowie ein Instrument zur Selbsteinschätzung digitaler Kompetenzen und stellt erste Validierungsergebnisse vor. 
Der Beitrag von Franco Rau und Ilaria Kosubski «Digitale Medien sind wie Pilze?» stellt Vorstellungen und Überzeugen von Lehramtsstudierenden als relevante Lernvoraussetzungen dar. Aufgrund dieser grossen Bedeutung für die Professionalisierung von Lehrpersonen wurde daher in einer empirischen Pilotstudie die Frage aufgeworfen, mit welchen Metaphern - als möglicher Zugang zu den Überzeugungen Lehramtsstudierende ihre Vorstellung von digitalen Medien beschreiben. Das Ergebnis sind fünf metaphorische Konzepte, welche im Beitrag näher vorgestellt werden.

Lisa Leander führt in ihrem Beitrag die Perspektiven der Wissenschaftskommunikation und des E-Learning zusammen und reflektiert die jeweiligen Perspektiven insbesondere im Hinblick auf informelles Lernen. Damit soll ein Ansatzpunkt geliefert werden, um Zielsetzungen und Vermittlungsmöglichkeiten von Lernangeboten im Kontext von Hochschulen zu analysieren. Anhand zweier Beispiele aus dem KIT veranschaulicht sie, wie Lernangebote gestaltet sein können.

Der Beitrag von Sarah Stumpf verfolgt das Ziel das Verhältnis von digitaler und sprachlicher Kompetenzen näher zu erläutern und skizziert die sprachlichen Herausforderungen, welche mit dem digitalen Leben einhergehen. Weiterhin werden Impulse hinsichtlich der Gestaltung und Operationalisierung eines Kompetenzmodells sprachlichen Handelns aufgezeigt.

Svenja Noichl stellt das wichtige Thema der Informatik-Kompetenz für technikferne Erwachsene vor, die so zur Teilhabe befähigt werden sollen. Hierfür wurden die Richtlinien für Schulen und aktuell interessante Themen in Deutschland beleuchtet sowie über eine Befragung von 123 Personen diejenigen Kompetenzen zu ermittelt versucht, die technikferne Erwachsene erwerben sollten.

Am Karlsruher Institut für Technologie sollen in einem Projekt «akademische digitale Medienkompetenz» bei Studierenden gefördert werden. David Lohner, Thomas May und Adnan Seithe beschreiben die verschiedenen zu fördernden Kompetenzdimensionen und die damit verbundenen Lernziele, sowie Veranstaltungen in denen diese gefördert werden sollen. Im Sinne der offenen Bildung soll der erste Kurs als Open Educational Ressource angeboten werden und somit auch anderen Dozierenden die Möglichkeit geben damit die akademische digitale Medienkompetenz bei ihren Studierenden zu fördern.

Im Fokus des Beitrags von Marcella Haller, Franco Rau, Sophie Schaper und Naheela Ulrich steht die Entwicklung eines Vernetzungsmoduls «Medienpädagogik» für das Lehramtsstudium an der TU Darmstadt. In diesem Praxisbericht wird eine Matrix präsentiert, mit deren Hilfe eine transparente Zusammenarbeit und Vernetzung der Fach- und Bildungswissenschaft sowie der Fachdidaktiken nicht nur denkbar, sondern durchführbar ist.

Der Artikel von Ina Schiedermair untersucht die Akzeptanz von von E-LearningFormaten der Region Westpfalz. Mithilfe dieser digitalen Lehr- Lernarrangements soll gesellschaftliche Teilhabe ermöglicht werden und Chancen zur Partizipation an 
Hochschulbildung für verschiedene Zielgruppen bieten. Es wird schliesslich darauf fokussiert, mögliche ablehnende Haltungen zu identifizieren und hierzu passende Angebote zur Akzeptanzsteigerung anzudenken.

René Röpke stellt die Ergebnisse eines Reviews zu spiel-basierte Lernanwendungen und Serious Games für grundlegende Kenntnisse und Fähigkeiten im Bereich der IT-Sicherheit und Sicherheitspraktiken vor. Es zeigte sich, dass es nur wenige online verfügbare Anwendungen gibt, die Nutzerinnen und Nutzer nachhaltige Kenntnisse in den Bereichen vermitteln und dies ein Bereich ist, in dem Entwicklungspotenzial vorhanden ist.

\section{Danksagung}

Wir möchten an dieser Stelle abschliessend allen danken, ohne die dieses Themenheft und auch das JFMH 2018 nicht möglich gewesen wären. An vorderster Stelle danken wir natürlich allen Teilnehmenden am JFMH 2018 in Kaiserslautern - den Referentinnen und Referenten, Moderatorinnen und Moderatoren sowie allen Teilnehmenden vor Ort. Durch all eure Beiträge in verschiedenster Form habt ihr dafür gesorgt, dass auch 2018 wieder einmal gezeigt hat, dass das JFMH ein Format ist, dass fruchtbaren Austausch in einer gleichwohl produktiven wie angenehmen Atmosphäre schafft. Ein besonderer Dank sei hier für unsere Keynote-Speakerinnen und -Speakern ausgesprochen: Danke Patrick Bettinger, Claudia Bremer und Felix Fischer.

Dank gilt auch der Technischen Universität Kaiserslautern sowie dem Fachgebiet Pädagogik, die uns bei der Ausrichtung der Tagung unterstützt haben. Besonderer Dank sei hier an Prof. Dr. Mandy Schiefner-Rohs und Prof. Dr. Matthias Rohs gerichtet, die uns im Planungs-, Organisations- und Durchführungsprozess stets unterstützend zur Seite gestanden und uns die Tagung nach unseren Vorstellungen haben gestalten lassen.

Bei unserer Kollegin Constanze Reder und unserem Kollegen Arash Tolou, die vor Ort mit uns die Tagung organisiert haben, möchten wir uns an dieser Stelle noch einmal für die gute Zusammenarbeit bedanken: es hat uns grossen Spass gemacht das JFMH mit euch zusammen auszurichten! Auch unseren studentischen Hilfskräften sei hier ein grosser Dank ausgesprochen - ohne euch hätte die Tagung nicht stattfinden können: Vielen lieben Dank Jennifer Bender, Jennifer Bloise, Isabelle Fiedler, Anna Herrmann, Philipp König, Jonathan Kohl, Christian Layes, Annika Lesch, Christian Scherner und Ina Schiedermair.

An dieser Stelle seien auch Janina Grabow und das Team vom eTeaching Service Center der TUK dankend genannt, die uns bei der Unterstützung des Videodreh und Schnitt zum "Teaser-Video" des JFMHs unterstützt haben.

Ohne die Reviewerinnen und Reviewern im Peer-Review-Prozess, sowohl bei der Vorbereitung der Tagungsbeiträge als auch der Beiträge in diesem Tagungsband, 
wäre das JFMH nicht das, was es ist. Namentlich sei gedankt: Patrick Bettinger, Valentin Dander, Nina Grünberger, Christiane Hagedorn, Anna-Lena Hebel Klaus Himpsl-Gutermann, Sandra Hofhues, Anna-Maria Kamin, Paul-Thomas Kandiza, Cornelia Kenneweg, Johannes Konert, Anja Lorenz, Agathe Merceron, Marianne Merkt, Anke Pfeiffer, Hans-Martin Pohl, Gisela Prey, Franco Rau, Christoph Rensing, René Röpke, Wolfgang Ruge, Peter Salden, Michael Schön, Antonia Scholkmann, Isabel Steinhardt, Angelika Thielsch, Alexandra Totter, Timo van Treeck, Jan Vanvinkenroye und Lorenz Wagner.

Allen Unterstützerinnen und Unterstützern darüber hinaus sei an dieser Stelle ebenfalls gedankt.

Ein grosser Dank geht natürlich auch an die einzelnen Fachgesellschaften - die dghd, die GMW, die E-Learning-AG der GI sowie die Sektion Medienpädagogik der DGfE und ihre jeweiligen Vertreterinnen und Vertreter. Wir danken für die beratende Unterstützung im Zuge der Vorbereitung und Durchführung des JFMHs und auch für die finanzielle Unterstützung.

Abschliessend möchten wir uns bei Klaus Rummler als Herausgeber der Zeitschrift MedienPädagogik bedanken, der uns im gesamten Prozess der Entstehung dieses Heftes stets beratend und unterstützend zur Seite Stand: Herzlichen Dank!

Nun wünschen wir allen Leserinnen und Lesern eine informative und unterhaltsame Lektüre der Beiträge in diesem Themenheft.

Die Herausgeber*innen

Kaiserslautern, November 2019 